\title{
A Comparison of SAME Slope Seasonality and Holt- WINTERS SMOOTHING FORECASTING MODELS
}

\author{
Hadis Bajric, Edin Kadric, Mugdim Pasic \\ Mechanical Engineering Faculty, University of Sarajevo, Vilsonovo šetalište 9, 71000 Sarajevo, \\ Bosnia and Herzegovina
}

\begin{abstract}
In this paper analytical expression for determining the optimal parameter value of the Same Slope Seasonality model was developed. Then performance of the Same Slope Seasonality model was compared to the performance of Holt-Winters exponential model, performing tests on M2-Competition time series. To determine the parameters of Holt-Winters exponential model, nonlinear mathematical programming was used. Performed tests proved that Same Slope Seasonality model is more successful than Holt-Winters exponential model. Tests revealed that Same Slope Seasonality model gives unreliable forecasts when time series has specific characteristics, giving us valuable information for model improvement.
\end{abstract}

Keywords: forecasting; Holt-Winters model; exponential smoothing; same slope seasonality model; time series; M2competition
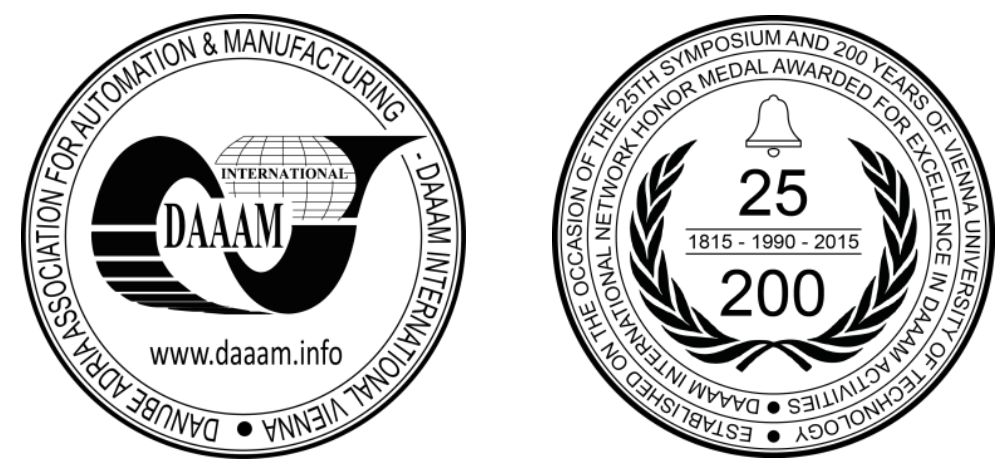

This Publication has to be referred as: Bajric, H[adis]; Kadric, E[din] \& Pasic, M[ugdim] (2016). A Comparison of Same Slope Seasonality and Holt-Winters Smoothing Forecasting Models, Proceedings of the 26th DAAAM International Symposium, pp.0062-0068, B. Katalinic (Ed.), Published by DAAAM International, ISBN 978-3-90273407-5, ISSN 1726-9679, Vienna, Austria DOI:10.2507/26th.daaam.proceedings.009 


\section{Introduction}

Forecasting model reliability is not conditioned by its complexity, and simple forecasting models can be accurate or even more accurate than more complex models [1]. According to [2] increasing complexity leads to reduced accuracy regardless of forecasting method used. In the works which were the subject of the study in [2], and where the increase in errors could be quantified, authors report that an increase in complexity increases the prediction error for about $25 \%$. Authors recommend that forecasters should use those forecasting models that they can understand.

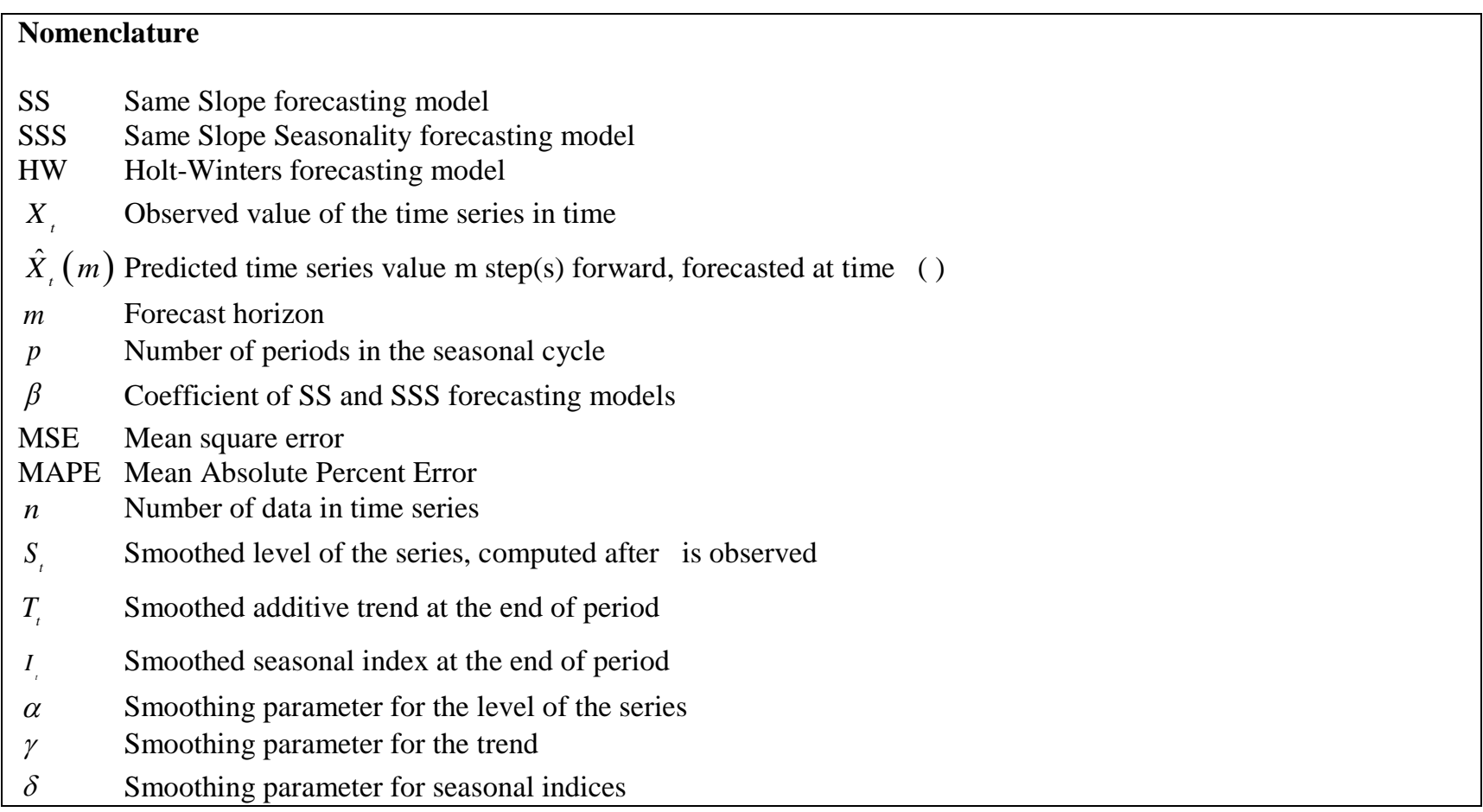

Exponential smoothing models because of their characteristics have found very wide application in production and service organizations [1,3]. Some authors because of their characteristics such as simplicity, ease of use and very good reliability, highlight these families of forecasting models even in relation to more complex such as ARIMA models $[4,5]$.

This paper deals with Same Slope (SS) and Same Slope Seasonality (SSS) forecasting models. SS and SSS forecasting models are of recent. In comparation to referent exponential smoothing models, SS and SSS are simpler models. Also, SS and SSS forecasting models are according to conducted studies [6, 7, 8] give forecasts of better reliability compared to exponential smoothing models.

Aim of this paper is to try to make SS and SSS forecasting models even easier to implement. In this paper, analytical expression for determining the optimal value of forecasting model parameter was developed. This work is an attempt to make SSS model even easier in terms of implementation. The existence of analytical expression for determining the optimal parameter value now does not require any minimization of the standard deviation of the forecasting model. Determining optimal forecasting model parameter value has so far required defining and solving of the nonlinear mathematical programming model.

In this study we also compared performance of the SSS model to the performance of Holt-Winters (HW) exponential forecasting model. To compare performances HW forecasting model is chosen as very popular model among practitioners and researchers [9]. Tests were performed on the time series of M2-Competition [10].

Settings of SS forecasting model are presented in [7]. In the same study, tests were carried out on the real time series. SS model is a single parameter model, simple to understand and apply. In [8] tests and performance comparation of the SS model to fifteen models of exponential smoothing have been performed. The results showed that the developed model is more efficient than models of the same level of complexity.

Main settings and results of performed tests of SSS forecasting model are presented in [6]. SSS forecasting model is still the single parameter model based on the same logic as SS model. The results showed that the developed model generates forecasts of the same level of reliability as well as more complex exponential smoothing models.

\section{Settings of the same slope and same slope seasonality models}

SS model is based on idea that time series will have the same gradient (slope) in the next time period like in previous. Model is defined by (1), whose settings are presented in [7]: 


$$
\hat{X}_{t}(1)=X_{t}-\beta\left(X_{t-1}-X_{t}\right)
$$

Graphical presentation of logic of SS model is given on Fig. 1.

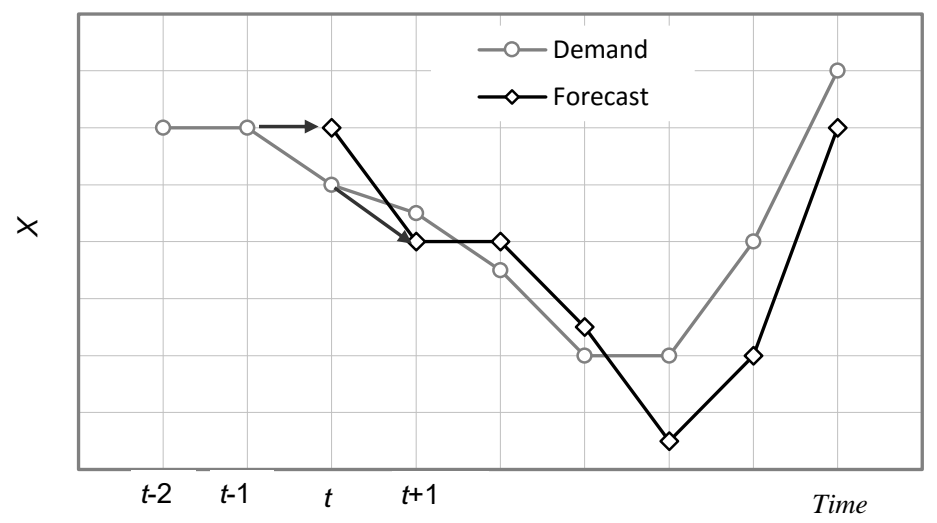

Fig. 1. Graphical presentation of logic of SS model

SSS model is based on the same idea that change in time series between $t$ and $t+m$ will be the same to the change time series had in time period between $t-p$ and $t-p+m$. Graphical presentation of logic of SSS model is given on Fig. 2.

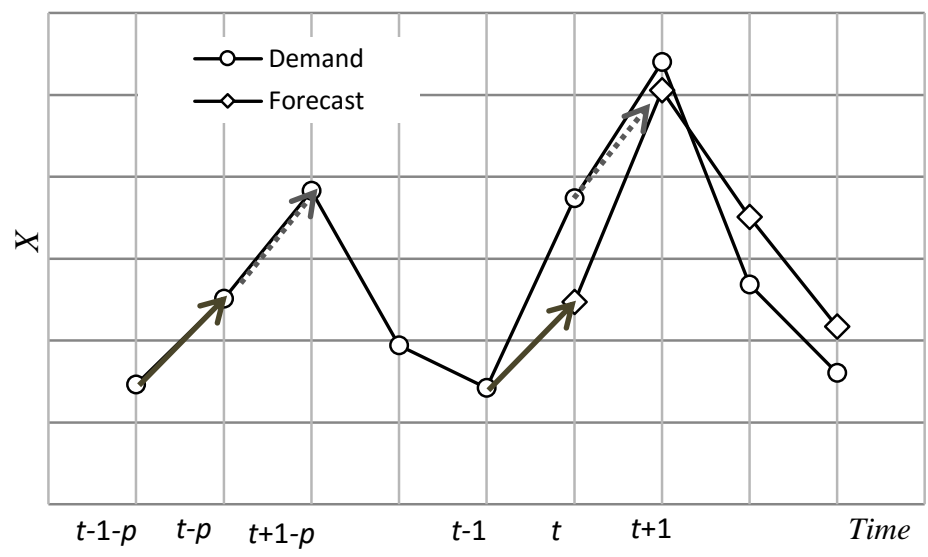

Fig. 2. Graphical presentation of logic of SSS model

SSS model is also single parameter forecasting model defined by (2), whose settings are presented in [6]:

$$
\hat{X}_{t}(m)=X_{t}-\beta\left(X_{t-p}-X_{t-p+m}\right)
$$

With respect to parameter $\beta$ following cases can be considered:

$\beta<1$ - forecast smaller change rate in the period $t$ to $t+m$ than in the prior period, e.g. forecasts smaller change rate in the period $t$ to $t+m$ than in the period $t-p$ to $t-p+m$.

$\beta=1$ - forecast the same change rate in the period $t$ to $t+m$ as in the period $t-p$ to $t-p+m$.

$\beta>1$ - forecast bigger change rate in the period $t$ to $t+m$ than in the period $t-p$ to $t-p+m$.

Considering (1) and (2) it can be concluded that in the case $p=1$, SSS model becomes SS model. Because of this fact, only analytical expression for determining parameter $\beta$ of SSS model is developed. 


\section{Determining optimal value of the same slope seasonality model parameter}

SSS model because of its mathematical simplicity, allows finding expression for determining optimal $\beta$ parameter. It is necessary to formulate function of squared deviations between forecasted and true values, dependent on $\beta$ parameter is given by:

$$
f(\beta)=\sum_{t=p+1}^{n+m-1}\left[X_{t+m}-\hat{X}_{t}(m)\right]^{2}
$$

Substituting (2) into (3) we get expression for sum of squared errors as the function of $\beta$ parameter:

$$
f(\beta)=\sum_{t=p+1}^{n+m-1}\left[X_{t+m}-X_{t}-\beta\left(X_{t-p}-X_{t-p+m}\right)\right]^{2}
$$

Function $f(\beta)$ is convex and its minimum can be found by equaling its first derivative to zero. The first derivative of the function of squared errors is given by:

$$
\frac{\partial f(\beta)}{\partial \beta}=\sum_{t=p+1}^{n+m-1} 2 \cdot\left[X_{t+m}-X_{t}+\beta\left(X_{t-p}-X_{t-p+m}\right)\right] \cdot\left(X_{t-p}-X_{t-p+m}\right)
$$

Equaling the first derivative of $f(\beta)$ function to zero gives analytical expression for calculating $\beta$ parameter:

$$
\beta=\frac{\sum_{t=p+1}^{n+m-1}\left(X_{t}-X_{t+m}\right) \cdot\left(X_{t-p}-X_{t-p+m}\right)}{\sum_{t=p+1}^{n+m-1}\left(X_{t-p}-X_{t-p+m}\right)^{2}}
$$

Where $n$ is number of data in time-series.

The value of $\beta$ parameter calculated by (6) corresponds to the value determined using nonlinear mathematical programming, where standard deviation or MSE of model is minimized.

\section{Research methodology}

Performances of SSS model are compared to the performances of three-parameter HW exponential smoothing model with additive trend and additive seasonal component, which is listed in standard list of exponential smoothing models (1):

$$
\left.\begin{array}{c}
S_{t}=\alpha\left(X_{t}-I_{t-p}\right)+(1-\alpha)\left(S_{t-1}+T_{t-1}\right) \\
T_{t}=\gamma\left(S_{t}-S_{t-1}\right)+(1-\gamma) T_{t-1} \\
I_{t}=\delta\left(X_{t}-S_{t}\right)+(1-\delta) I_{t-p} \\
\hat{X}_{t}(m)=S_{t}+m T_{t}+I_{t-p+m}
\end{array}\right\}
$$

Tests are performed on times series "M2CAll" from M2-Competition, available at [11]. Last year data have been used to estimate MAPE of the considered models, and all other data are used to estimate optimal values of forecasting models parameters.

SSS model parameter is determined using (6) for case $m=1$. To determine HW model parameters, nonlinear mathematical programming is used. Nonlinear mathematical programming models are solved using Solver in Microsoft Excel application. The model standard deviation is used as the objective function of nonlinear mathematical programming model, also for the case $m=1$.

Forecasts are generated for time horizon of one year. After forecasts are generated, true time series values are used to estimate reliability measures. To compare model performances MAPE is used as a measure of model reliability. Forecasts are generated using only quantitative data and without any available qualitative informations. 
Tests are performed on 28 of 29 time series from M2-Competition. Time series INTERSAL haven't been used in tests, because its value in the last year is 0 , which prevents calculating MAPE value. Among 28 analyzed, 22 were monthly and 6 quarterly time series.

\section{Results}

The developed expression for determination of SSS model $\beta$ parameter, for each of the tested time series, gives $\beta$ value identical to the value obtained by using Solver in MS Excel, set to solve nonlinear programming model in a way that the problem is set up to search the $\beta$ parameter which minimizes the standard deviation of the model.

MAPE values for both models and all analyzed time series are given in Table 1. It can be seen from Table 1. that SSS model has better performances for 17 time series, while HW model has for 11. Average MAPE value for SSS model is $25,56 \%$ and $24,03 \%$ for HW model. If three time series (PANTER, PHARMA11 and TRADUSAL), for which both models have high uncertainty levels, are omitted from analysis, then average MAPE values are 11,80 and 12,41\%, respectively.

\begin{tabular}{lll}
\hline Series & HW model & SSS model \\
\hline PANTER & $284,54 \%$ & $259,65 \%$ \\
CHEETAH & $18,39 \%$ & $17,43 \%$ \\
LION & $29,45 \%$ & $37,15 \%$ \\
BIGCAT & $13,50 \%$ & $15,34 \%$ \\
ANIMAL & $14,47 \%$ & $16,94 \%$ \\
CARINDS & $13,03 \%$ & $12,85 \%$ \\
REALGNP & $0,45 \%$ & $7,06 \%$ \\
IPDGNP & $0,17 \%$ & $0,79 \%$ \\
IPDIMP & $2,55 \%$ & $0,97 \%$ \\
FIXINV & $4,60 \%$ & $4,43 \%$ \\
COMPIND & $1,44 \%$ & $0,35 \%$ \\
BUSINV & $10,24 \%$ & $7,48 \%$ \\
OEMUSAL & $18,00 \%$ & $10,32 \%$ \\
TRADUSAL & $36,32 \%$ & $58,16 \%$ \\
FLMARKT & $22,54 \%$ & $8,26 \%$ \\
RESDVSAL & $10,87 \%$ & $7,79 \%$ \\
INDSHIPF & $10,49 \%$ & $9,30 \%$ \\
PHARMA11 & $41,73 \%$ & $102,92 \%$ \\
PHARMA22 & $30,04 \%$ & $21,50 \%$ \\
PHARMA33 & $28,48 \%$ & $23,59 \%$ \\
METOGEN & $24,25 \%$ & $23,64 \%$ \\
CARDERS & $23,23 \%$ & $22,40 \%$ \\
NEWRX & $3,66 \%$ & $2,97 \%$ \\
REFILLS & $4,32 \%$ & $2,73 \%$ \\
UNCPAPEUR & $7,08 \%$ & $18,37 \%$ \\
CPAPEUR & $8,59 \%$ & $10,21 \%$ \\
UNCPAPUSA & $5,34 \%$ & $6,99 \%$ \\
CPAPUSA & $5,18 \%$ & $6,17 \%$ \\
\hline
\end{tabular}

Table 1. Comparative display of MAPE values for all analyzed time-series

It can be concluded, based on test results shown in Table 1, that SSS forecasting model has shown better performances than more complex HW forecasting model on respectable number of time series. Important advantage of SSS in relation to HW forecasting model is existence of analytical expression for direct calculation of optimal $\beta$ parameter value. For estimation of optimal parameter values of HW model it is necessary to use nonlinear mathematical programming and appropriate solver applications. Importance of developed model is even bigger if it is known that HW forecasting model is very robust and reliable, which in many studies shows close or better performances even than very complex ARIMA models [1, 12, 13, 14]. 


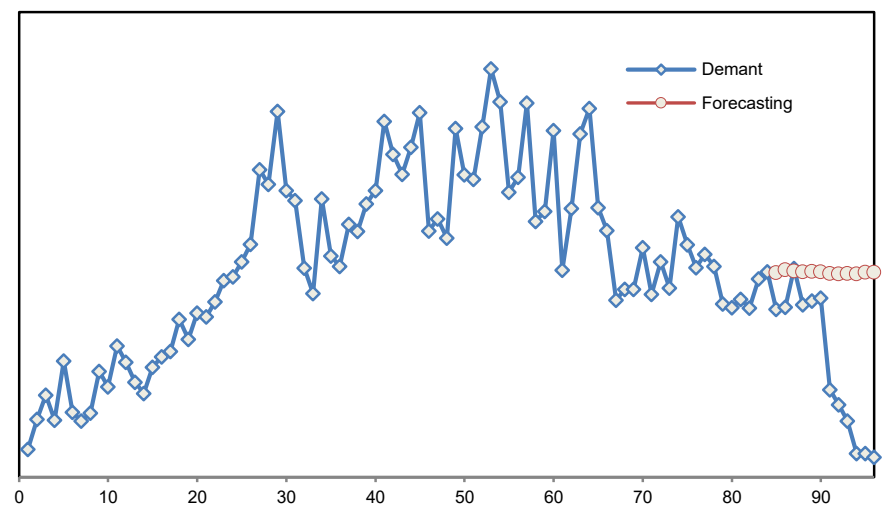

Fig. 3. PANTER Time series

While performing tests, we discovered very important disadvantage of SSS model, and it is that forecast results are very affected by last data in time series. This disadvantage is not present when time series has no non-standard observations. Model gives very poor results in case that last available data in time series is non-standard observation. As an example of unreliable forecast is forecast of PANTER time series, where SSS model had MAPE value of $259,65 \%$. Poor forecast results do not mean model is unreliable, because for the same time series HW model gave even poorer results. It is obvious that random component is very dominant in this time series, and in order to do meaningful forecasts, some qualitative informations are required.

\section{Conclusion}

The developed expression for determination of SSS model $\beta$ parameter, for each of the tested time series, gives $\beta$ value identical to the value obtained by using Solver in MS Excel, set to solve nonlinear programming model in a way that the problem is set up to search the $\beta$ parameter which minimizes the standard deviation of the model.

SSS model has shown better results than HW model in the tests performed on 28 M2-Competition time series. SSS model had less MAPE value on 17, while HW model had on 11 time series.

SSS model is single parameter model having only one expression, while HW model is three-parameter model having three expressions, required for determination of model parameters.

Comparative advantage of SSS model to HW model especially is important when considering costs of parameter determination. SSS model parameter is simply determined using single analytical expression, while HW model parameter determination requires solving nonlinear mathematical programming model. Besides this obvious advantage, SSS model is easier to understand and implement than HW model, and also it requires less computational operations and efforts in forecast generations. Forecasts generated by SSS model are of the same reliability levels as forecasts generated by more complex HW model.

SSS model performed well in forecasting time series with dominant seasonal as well as trend component. Unreliable forecasts by SSS model can be expected in case of non-standard observations in the time series, because SSS model forecasts are very dependent on current observation for which forecasting value is generated.

By introducing another parameter, which should relativize influence of single observation, maybe would improve disadvantage of SSS model that one last observation has such serious impact on forecast. Another suggestion is that instead of using value of one observation, value of moving average for some period length could be used.

\section{References}

[1] S. Makridakis, M. Hibon, The M3-Competition: results, conclusions and implications. International Journal of Forecasting, 16 (2000) 451-476.

[2] C. K., Green, J. S. Armstrong, Simple versus complex forecasting: The evidence. Journal of Business Research, 68 (8) (2015) 1678-1685.

[3] E. S. Gardner, Exponential smoothing: The state of the art - Part II, International Journal of Forecasting, 22 (4) (2006) 637-666.

[4] R. L. Paul, D.John, L Geoff, Moving towards a control technique to help small firms monitor and control key marketing parameters: a survival aid, Management Decision, 39 (2) (2001) 113-120.

[5] C. Chatfield, The Analysis of Time Sries: An Introduction, Chapman and Hall, London, 1989.

[6] H. Bajrić, I. Bijelonja, M. Pašić, A Comparison of Same Slope Seasonality and Exponential Smoothing Forecasting Models, Proceedings of the 20th International DAAAM Symposium, B. Katalinic, (Ed.), (2009) 13431344.

[7] M. Pašić, I. Bijelonja, A. Šunje, H. Bajrić, (2007). Same Slope Forecasting Method, Proceedings of the 18th International DAAAM Symposium, B. Katalinic, (Ed.), (2007) 547-548. 
[8] M. Pašić, I. Bijelonja, H. Bajrić, A Comparison of Same Slope and Exponential Smoothing Forecasting Models, Proceedings of the 19th International DAAAM Symposium, B. Katalinic, (Ed.), (2008) 516-517.

[9] J. W. Taylor, Exponential Smoothing with a Damped Multiplicative Trend, International Journal of Forecasting, 19 (2003) 715-725.

[10] S. Makridakis, C. Chatfield, M. Hibon, M. Lawrence, T. Mills K. Ord, L. F. Simmons, The M2-Competition: A real-time judgmentally based forecasting study, International Journal of Forecasting, 9 (1993) 5-22.

[11] IIF, International Institute of Forecasters, M2-Competition, Available from: http://forecasters.org/resources/timeseries-data/m2-competition/ Accessed: 2015-05-30.

[12] D. Puthran, H. C. Shivaprasad, K. S. Keerthesh Kumar, M. Manjunath, Comparing SARIMA and Holt-Winters' forecasting accuracy with respect to Indian motorcycle industry, Transactions on Engineering and Sciences 2 (5) (2014).

[13] K. Tanvir, Identifying an Appropriate Forecasting Model for Forecasting Total Import of Bangladesh, International Journal of Trade, Economics and Finance, 2 (3) (2011).

[14] Walters, Q. Cai, Investigating the use of holt-winters time series model for forecasting population at the state and sub-state levels. Demographics and Workforce Section,Weldon Cooper Center for Public Service, University of Virginia, 2008. 